\title{
The effect of a pressure vest on the behaviour, salivary cortisol and urine oxytocin of noise phobic dogs in a controlled test
}

\section{Pekkin, Anne-Marie}

2016

Pekkin , A-M , Hänninen , L, Tiira , K, Koskela , A , Pöytäkangas , M , Lohi , H \& Valros , A 2016 , ' The effect of a pressure vest on the behaviour, salivary cortisol and urine oxytocin of noise phobic dogs in a controlled test ' , Applied Animal Behaviour Science, vol. 185 , pp. 86-94 . https://doi.org/10.1016/j.applanim.2016.09.003, https://doi.org/10.1016/j.applanim.2016.09.003

http://hdl.handle.net/10138/231340

https://doi.org/10.1016/j.applanim.2016.09.003

publishedVersion

Downloaded from Helda, University of Helsinki institutional repository.

This is an electronic reprint of the original article.

This reprint may differ from the original in pagination and typographic detail.

Please cite the original version. 


\title{
The effect of a pressure vest on the behaviour, salivary cortisol and urine oxytocin of noise phobic dogs in a controlled test
}

\author{
Anne-Maria Pekkin ${ }^{a}$, Laura Hänninen ${ }^{\text {b, }}$, Katriina Tiira ${ }^{\mathrm{d}}$, Aija Koskela ${ }^{\mathrm{b}}$, \\ Merja Pöytäkangas ${ }^{\mathrm{b}}$, Hannes Lohi ${ }^{\mathrm{c}}$, Anna Valros $^{\mathrm{b}}$ \\ a Clinic of Child Psychiatry, PEDEGO Research Unit, University of Oulu, Finland \\ ${ }^{\mathrm{b}}$ Faculty of Veterinary Medicine, University of Helsinki, Finland \\ ${ }^{\mathrm{c}}$ Department of Veterinary Biosciences and Research Programs Unit, Molecular Neurology, University of Helsinki, Finland \\ ${ }^{\mathrm{d}}$ The Folkhälsan Research Center, Helsinki, Finland
}

\section{A R T I C L E I N F O}

\section{Article history:}

Received 25 April 2016

Received in revised form 31 August 2016

Accepted 11 September 2016

Available online 14 September 2016

\section{Keywords:}

Dog

Pressure vest

Noise phobia

Cortisol

Oxytocin

Behaviour

\begin{abstract}
A B S T R A C T
Fear of loud noises is a common welfare problem in pet dogs. Commercial treatment vests have been tested on dogs to relieve noise phobia, and peripheral oxytocin has been suggested to be one of the stressrelieving mediators. The effect of vests has not, however, been tested in a controlled situation. We tested whether individually customized vests, have an effect on behaviour of severely noise phobic dogs in a double-blinded experiment, where dogs are exposed to loud noises. We also investigated the possible effect of pressure by using two types of vests; a deep pressure vest (DEEP, c. 10-12 $\mathrm{mmHg}$ ) and a light pressure vest (LIGHT, c. $2-3 \mathrm{mmHg}$ ). In addition to behaviour, we studied if the pressure vest has an effect on urine oxytocin level and on saliva cortisol levels.

A total of 28 dogs (2-11 years), both female (18) and male (10), were recruited via an ongoing study on the genetic background of noise sensitivity by the Finnish Canine Genetic Research Group. Each dog was tested three times either without vest (CONTROL) or with DEEP or LIGHT vests in a semi-randomized order. The dogsí behaviour was video recorded for $6 \mathrm{~min}$, including three $2 \mathrm{~min}$ intervals: pre-noise, noise (70-73 dB firework sound) and recovery. Behavioural parameters included activity, body and tail postures, vocalization, and time spent near owner. Saliva samples were collected twice before and twice after the noise test. Urine samples for oxytocin analyses were collected when the deep pressure vest was first fitted: before dressing the dog and 30 min later.

The DEEP vest reduced the lying time of the dogs during the noise interval. During the noise interval total lying time with any vest correlated positively with saliva cortisol, measured after noise interval, indicating that the increased lying time was a sign of a higher stress level in the dogs. Wearing either vests increased the time the dogs spent near their owners during noise interval. Time spent near the owner when wearing the DEEP vest during the recovery interval correlated positively with the urine oxytocin. These results indicate oxytocin might be related to the dogís tendency to seek owner support and the vests might effect this behaviour positively. We did not find a clear therapeutic effect of using pressure vests in noise phobic dogs. However, our results indicate the pressure vest might reduce the acute stress reaction and speed up the recovery after stress.
\end{abstract}

(C) 2016 Elsevier B.V. All rights reserved.

\section{Introduction}

Noise phobia, or sensitivity, is a very common welfare problem in pet dogs (Sherman and Mills 2008; Blackwell et al., 2013): up

\footnotetext{
* Corresponding author.

E-mail addresses: Anne.Pekkin@student.oulu.fi (A.-M. Pekkin), laura.hanninen@helsinki.fi (L. Hänninen), katriina.tiira@helsinki.fi (K. Tiira), aija.koskela@helsinki.fi (A. Koskela), merja.poytakangas@helsinki.fi (M. Pöytäkangas), hannes.lohi@helsinki.fi (H. Lohi), anna.valros@helsinki.fi (A. Valros).
}

to $50 \%$ of dog owners have reported their pets to be afraid of at least one type of noise (Blackwell et al., 2013). Typically dogs react aversively to thunder, fireworks and shotguns. If the dog reacts fearfully towards one of these noise types, it has a high likelihood of reacting fearfully also to the other two noise types (Blackwell et al., 2013; Tiira et al. in Press). It is a general belief that noise phobia is associated with traumatic experiences, such as exposure to unexpected fireworks, however this is rarely the case (Overall 2000, 2013; Sherman and Mills, 2008). Noise sensitivity is suggested to have a clear hereditary component (van der Waaij et al., 2008) but also several environmental factors effect the development of the 
phobia (Blackwell et al., 2013; Overall, 2013; Tiira and Lohi, 2015). Dogs of all ages can suffer from noise phobia, while it gets more common with increasing age of the dog (Blackwell et al., 2013; Mills 2005; Tiira and Lohi, 2015). In a recent study, the average age for the onset of noise sensitivity was two years (Tiira and Lohi, 2015). Noise phobia is treated using de-sensitizing, pheromones, medication, and their combination, with variable success (Sherman and Mills, 2008).

The most common behavioural signs of noise fear are panting, trembling, hiding, escaping, restless walking and vocalizing. In extreme cases the dog may also urinate or defecate indoors (Blackwell et al., 2013; Dale et al., 2010; McCobb et al., 2001). Loud noise has further been shown to cause several physiological reactions in noise phobic dogs: increased heart rate and hematocrit level as well as increased plasma concentrations of cortisol, progesterone, vasopressin, and endorphins (Hydbring-Sandberg et al., 2004).

In clinical studies of humans, deep pressure and weighted vests or blankets have been shown to have a calming effects (Blairs et al., 2007; Edelson et al., 1999; Grandin, 1992; Mullen et al., 2008). When cattle were being held in a squeeze chute, which causes large area pressure, agitation was reduced in some animals (Grandin, 1992). In dogs suffering from separation anxiety pressure vests have been shown to reduce heart rate (King et al., 2014). Commercial pressure vests have been tested also on dogs suffering from thunderstorm phobia. Some of the owners reported the use of the pressure vest to be successful (Cottam et al., 2013). However the effect of the vest has not been tested in a controlled situation and the possible underlying biological mechanism is still unknown.

Peripheral oxytocin has been suggested to be one of the mediators in the stress-relief action of skin contact (Bello et al., 2008) and effectively reduces blood pressure and heart rate (Pedersen et al., 2000). Oxytocin is also important for social attachment (Carter 2003; Panksepp 1992; Pedersen et al., 1988; Uvnäs-Moberg, 2006). In dogs, positive interactions with owners have been shown to increase the blood oxytocin level (Odendaal and Meintjes, 2003).

The aim of this study was to investigate the effect of pressure on behaviour of severe noise phobic dogs, and to investigate the possible role of oxytocin in mediating such an effect. We tested vests of two different pressure levels in a controlled test situation. We hypothesized that an individually customized vest with high pressure (DEEP) would reduce fear-related behaviours and saliva cortisol concentration of noise phobic dogs in a noise test compared to the (LIGHT) pressure vest, or no vest (CONTROL). We also expected to see an increase in the dog urine oxytocin after being exposed for $30 \mathrm{~min}$ to the deep pressure vest.

\section{Materials and methods}

The study was approved by the ethics committee of the University of the Helsinki in Finland.

\subsection{Experiment animals}

In total 28 dogs were recruited via an ongoing study by the Canine Genetic Research Group of the University of Helsinki, which investigates the genetic background of noise sensitivity. Owners had completed an online questionnaire about their dogs. The questionnaire included several fear-related questions, such as possible fearful reactions towards loud noises (thunder, fireworks and gunshot), unknown people, dogs or new situations. The questionnaire has been shown to have a good external validity and also excellent test-retest reliability especially in questions of the fearful reactions towards loud noises (Tiira and Lohi, 2014).

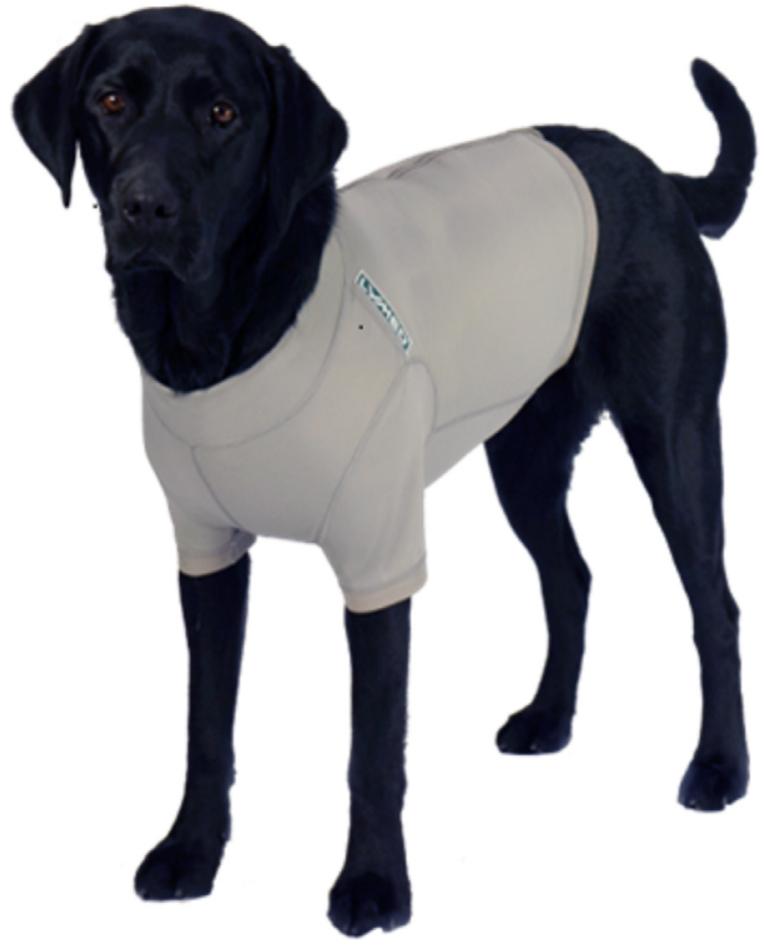

Fig. 1. The tested commercial deep pressure vest, Lymed Animal ${ }^{\mathrm{TM}}$ supporting garments, Lymed Ltd $^{\circledR}$., Finland.

All participating dogs (age 2-11 years, mean 5.9 years) had been reported to react fearfully in $60-100 \%$ of the occasions when exposed to fireworks, by either escaping or hiding. To avoid that the dog's possible fear towards the tester or novel situation would confuse the test, we excluded dogs that had shown fearful behaviours towards people or in new situations frequently (defined as $40-100 \%$ of occasions). Female dogs in estrus and dogs using regular medication were excluded, except for dogs using non-steroidal anti-inflammatory drugs.

The participating dogs represented 14 breeds, where Lagotto Romagnolo $(\mathrm{N}=7)$ and Staffordshire Bullterriers $(\mathrm{N}=6)$ were the most frequent breeds. Of the dogs 18 were bitches (including 14 sterilized) and 10 males (including 5 castrated). Owners reported that 22 out of the 28 dogs had used some form of clothes (e.g. raincoats and warm dog coats) at least once before, and a commercial noise desensitizing $\mathrm{cd}$ had been tested on seven dogs in the past, however, without success.

\subsection{A pressure vest}

The vests (Lymed Animal ${ }^{\mathrm{TM}}$ supporting garments, Lymed Ltd $^{\circledR}$ Finland) used in the experiment (Fig. 1) were personally customized to each dog. Experienced persons took individual measures of each dog for the vests at least two weeks before the experiment started. Two vests of similar texture, but with different pressure level were used: The typical Lymed deep pressure vest (DEEP) created a pressure of approximately $10-12 \mathrm{mmHg}$ and the light pressure vest (LIGHT) a pressure of approximately $2-3 \mathrm{mmHg}$.

\subsection{Test setup}

Dogs were semi-randomly divided into three treatment order groups, balancing for gender (Table 1). Each dog participated in three noise test days (1st, 2nd or 3rd) with the minimum time between two test days being one week. Each dog thus underwent all three treatments (CONTROL, LIGHT, and DEEP). 


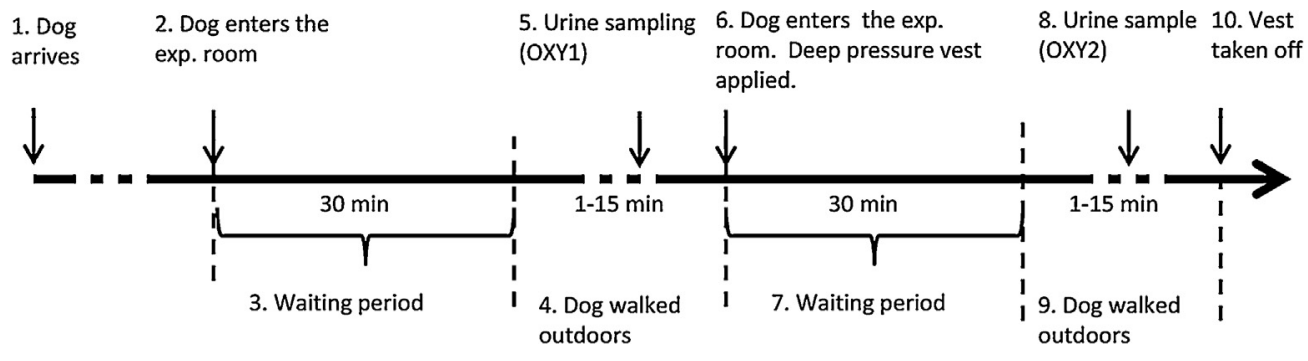

Fig. 2. The procedure of taking urine samples (oxytocin).

Table 1

Order of the tested vest treatments the DEEP pressure vest, the LIGHT pressure vest and CONTROL (without a vest) in a noise test. The DEEP pressure vest created a pressure of approximately $10-12 \mathrm{mmHg}$ and the LIGHT vest a pressure of approximately $2-3 \mathrm{mmHg}$.

\begin{tabular}{llll}
\hline Group & \multicolumn{3}{l}{ Order of test days, and number of participating dogs $(\mathrm{n})$} \\
\cline { 2 - 4 } & $\begin{array}{l}\text { 1st Test day } \\
(\mathrm{n}=28)\end{array}$ & $\begin{array}{l}\text { 2nd Test day } \\
(\mathrm{n}=26)\end{array}$ & $\begin{array}{l}\text { 3rd Test day } \\
(\mathrm{n}=25)\end{array}$ \\
\hline $1(\mathrm{n}=10)$ & LIGHT & CONTROL & DEEP \\
$2(\mathrm{n}=9)$ & DEEP & LIGHT & CONTROL \\
$3(n=9)$ & CONTROL & DEEP & LIGHT \\
\hline
\end{tabular}

Prior to each test the dogs had not been fed for at least an hour. Possible physiological treatments such as massage, acupuncture or trimming had been completed no later than one week before. At arrival the dog was walked on leash by the owner directly from the car to the examination room, without giving the dog any opportunity to sniff people or other dogs. The owners were advised to avoid any extra attention (such as petting and talking) towards their dog during any of the test phases. Test days were not organized during thunder.

\subsection{Urine samples and vest fitting}

Minimum of one week prior to the first test day the dogs and their owners arrived to the experiment room in the Veterinary Teaching Hospital (the University of Helsinki) for the DEEP vest fitting, urine sampling and adaptation to the experiment site. Dogs were not allowed to urinate at arrival. Two urine samples were collected for oxytocin analyses: one before fitting the vest (OXY1) and one after fitting and wearing the vest for $30 \mathrm{~min}$ (OXY2). To collect urine samples, the dogs were taken to an outside yard close to the experiment room. The dogs were kept on leash and urine samples were taken with a long-handled $1.5 \mathrm{dl}$ scoop. The samples were then placed on ice and transported to laboratory immediately after the 2nd sample (OXY2). The urine sampling schedule is shown in Fig. 2.

\subsection{The noise test and saliva sampling}

For the noise test, a $235 \times 245 \mathrm{~cm}$ area with a one-meter high fence was constructed within the experiment room (Fig. 3). The window of the room was covered. A small table was situated in the corner of the test arena, and it was covered with a cloth to give the dogs the possibility to hide underneath. For the behavioural scoring the floor was divided into 16 equal-sized squares. Loudspeakers for the noise stimuli were located in two opposite corners of the room. The owner was sitting on a chair next to the fenced area, facing the door. Researchers worked behind a visual barrier during the video recordings. Before the dog entered the room the noise level of the stimuli to be used was calibrated as $70-73 \mathrm{~dB}$ with a sound level meter (Nor131, Norsonic, Lierskogen, Norway). The same room was used throughout the study period (May to

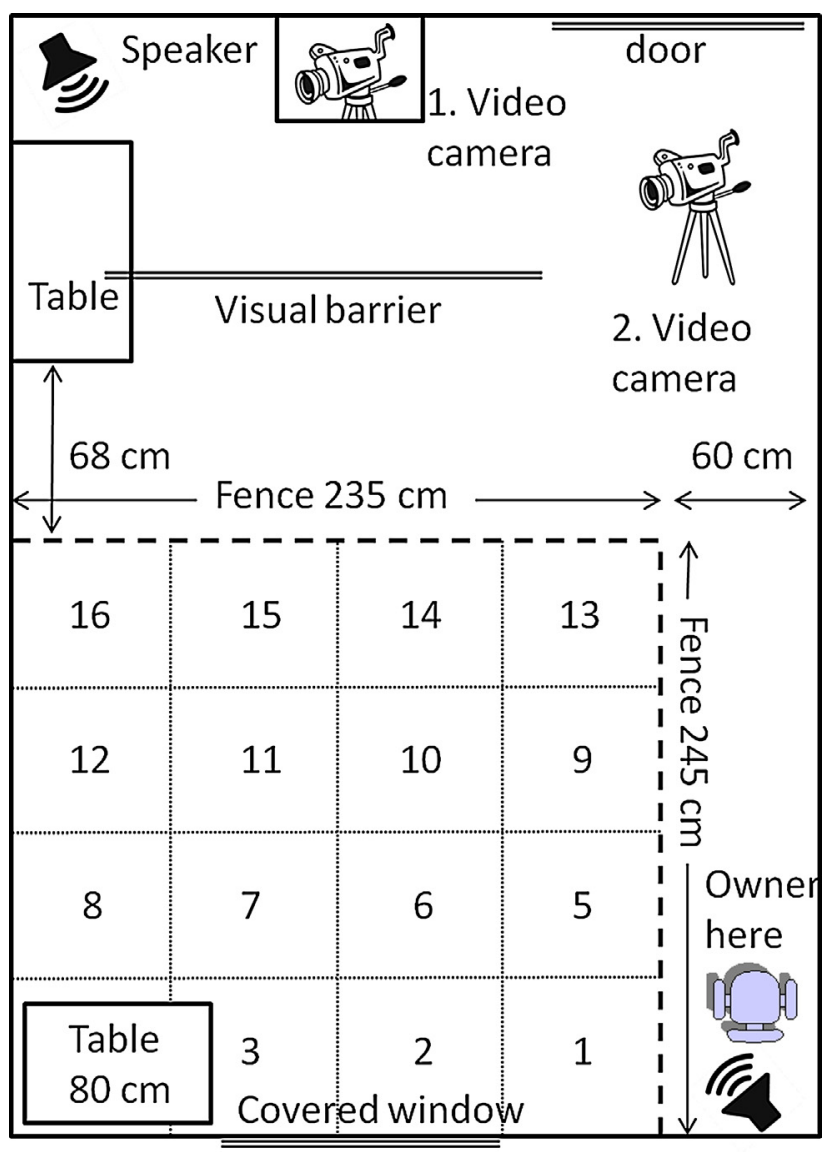

Fig. 3. The experiment set-up for the noise test. The second video camera was used as backup only.

September 2013). Between the tests sequences the room floor was cleaned for removing odours and providing appropriate hygiene (Kiilto Desichlor, KiiltoClean OY, Finland).

Saliva samples for cortisol analysis were collected using Salivette tubes (Cortisol-Salivette ${ }^{\circledR}$, Sarstedt AG \& Co, Nümbrecht, Germany), containing biodegradable synthetic swabs. In the morning of the test day the swabs were placed in a covered plastic jar, containing liverwurst for scenting. The swabs were not in physical contact with the liverwurst. The scenting was performed to increase saliva flow and to make sampling less stressful for the dogs. At sampling, the swab was taken from the jar using surgical forceps and placed in the dog's mouth. The dog was allowed to chew on the swab for 1-2 min, after which the swab was placed in a Salivette tube, placed in ice, and frozen as soon as possible after the test.

At arrival (Fig. 4) on the test days the dog was allowed to urinate before entering the experimental room (1). The first saliva sample (COR1) was taken outside at arrival (2). In the experimental room 
Table 2

Ethogram used for registration of dog behaviour. Behaviour was registered continuously throughout the 6 min test period.

\begin{tabular}{|c|c|c|}
\hline Behavioural classes & Behaviour & Definition \\
\hline Body postures & $\begin{array}{l}\text { Lying } \\
\text { Sitting } \\
\text { Standing }\end{array}$ & $\begin{array}{l}\text { Ventral/lateral body parts are touching the floor. } \\
\text { Hind quarters are touching the floor with the front legs being used for support. } \\
\text { All four paws touching the floor, legs upright and extended, supporting the body. Dog } \\
\text { is moving or standing still. }\end{array}$ \\
\hline \multirow[t]{3}{*}{ Motor activity } & Fence manipulation & $\begin{array}{l}\text { Touching fence with nose or front paws in such a way that fence is moving (i.e. } \\
\text { jumping against the fence/nosing it) }\end{array}$ \\
\hline & 1. Tail over the backline & a. \\
\hline & $\begin{array}{l}\text { 2. Tail along the backline } 3 \text {. Tail below the } \\
\text { backline } 4 \text {. Tail between legs } \\
5 \text {. Can't see the tail from video records. Dog is } \\
\text { sitting/standing back arched, head against the } \\
\text { camera. }\end{array}$ & \\
\hline \multirow[t]{2}{*}{ Location } & Location on the grid of squares & The square (1-16) where more than half of the dogís body is located (Fig. 3) \\
\hline & Location under the table & Registered when either at least half of the dogs body, or itsí head is under the table \\
\hline Vocalization & Vocalization by the dog & Any type of vocalization by the dog \\
\hline \multirow[t]{3}{*}{ Oral behaviours } & Yawn & $\begin{array}{l}\text { Dogs' mouth is stretching wide open for a period of a few seconds. Not related to } \\
\text { barking. }\end{array}$ \\
\hline & Lick nose or lips & $\begin{array}{l}\text { Tongue extends up- or sidewards to touch nose or lips, before retracting back into } \\
\text { mouth }\end{array}$ \\
\hline & Pant & $\begin{array}{l}\text { Mouth open with tongue extended accompanied with rapid breathing and chest } \\
\text { movements. If the dog's mouth was not visible, panting was scored from visible rapid } \\
\text { breathing movements of the chest. }\end{array}$ \\
\hline Other & Trembling & Dog shivering or trembling. \\
\hline
\end{tabular}

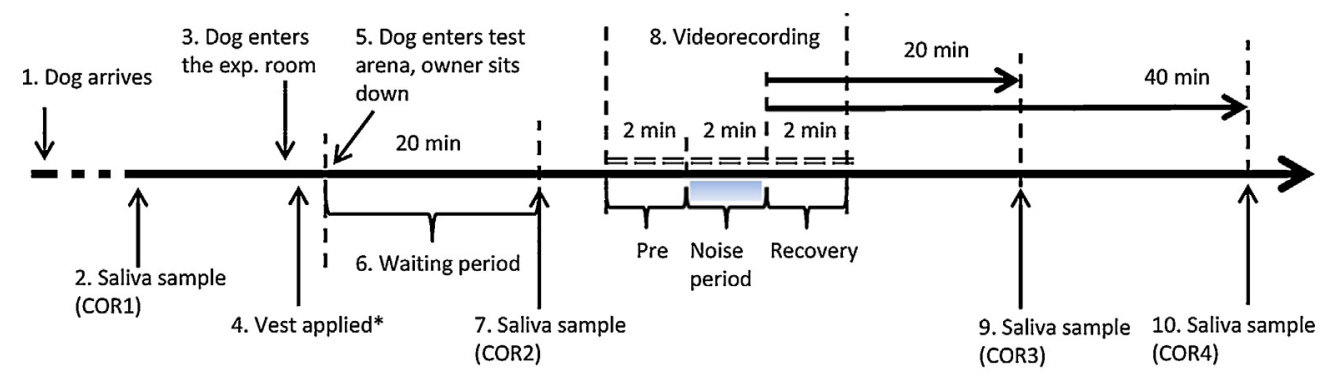

* In the control situation, the dog is touched in a way similar to when applying the vest

Fig. 4. The test set-up for the noise test.

the dog was dressed in the vest or if tested without the vest, the dog was touched in a similar way as if dressed in a vest (4). The owner was instructed to sit on the chair facing the door and asked not pay any attention to the $\operatorname{dog}(5)$. If the dog tried to get over the fence, the owner was told to prevent it with his/her hand.

Before the noise test began the dogs were given 20 min to adapt to the experiment room. At the end of the adaption period, a second cortisol (COR2) sample was taken (7). The test period was then started (8), and was divided into three two-minute intervals (prenoise interval, noise interval and recovery interval). The pre-noise interval was a quiet period without noise. The noise interval was a period of firework noise from loudspeakers played at 70-73 dB from a commercial cd aimed at desensitizing noise-phobic dogs (Hui Kauhistus - Sounds Scary, Sound Therapy 4 Pets, UK). The recovery interval was again a period without noise. Saliva samples COR3 and COR4 were taken $20 \mathrm{~min}$ (9) and $40 \mathrm{~min}$ (10) after the noise test (Fig. 4).

\subsection{Behaviour observations}

The behaviour of each dog was recorded throughout the 6 min test period with a video camera attached to a two meter high tripod above the test arena (Fig. 3). One person blinded to the treatments and aims of the study scored the activity (number of crossed lines), body and tail postures, oral behaviours and vocalizing continuously using the CowLog software (Hänninen and Pastell, 2009). Total duration, bout duration and number of bouts were calculated for body and tail postures, and number of bouts for panting, yawn- 
Table 3

Behavioural variables derived from the owner-filled questionnaire (Tiira and Lohi, 2014).

\begin{tabular}{ll}
\hline Variable & Explanation \\
\hline $\begin{array}{l}\text { Noise reactivity } \\
\text { index }\end{array}$ & $\begin{array}{l}\text { Describes the frequency and intensity of fearful } \\
\text { reactions towards loud noise. Calculated as follows: } \\
\text { (sum of fearful behavioural reactions to fireworks) }\end{array}$ \\
& $\begin{array}{l}\text { frequency of fear reaction to fireworks + (sum of fearful } \\
\text { behavioural reactions to thunder) }{ }^{*} \text { frequency of fear } \\
\text { reaction to thunder + (sum of fearful behavioural } \\
\text { reactions to gunshot })^{*} \text { frequency of fear reaction to } \\
\text { gunshot. }\end{array}$ \\
& $\begin{array}{l}\text { Sum of frequencies of showing fearful behaviour } \\
\text { towards loud noises (thunder, fireworks, gunshot). } \\
\text { Differs from noise reactivity index in that only the } \\
\text { frequency }\end{array}$ \\
& $\begin{array}{l}\text { frequency, but not the intensity is calculated. } \\
\text { Sum of frequencies of showing fearful behaviour } \\
\text { towards people, dogs, new situations }\end{array}$ \\
&
\end{tabular}

ing, licking, fence manipulation and crossed lines. The ethogram of recorded behaviours is shown in Table 2 .

\subsubsection{Owner surveys}

Prior to the experiment the dog owners had completed a survey about their dog's everyday behaviour. Several behavioural variables were derived from the questionnaire (Table 3), and these variables were used to investigate if the behaviour at home associates with the behaviour in the test situation.

After the 3rd and final noise test day the owners were allowed to take the deep pressure vest home. The first follow-up questionnaire was sent two months later in November. The owners were asked: Did you use the DEEP pressure vest on your dog in daily life? In what kind of situations? Was it helpful for your dog? The second followup questionnaire was sent just after the New Year. The owners were asked: Did you use the DEEP pressure vest on your dog at the New Year's eve? In what kind of situation? Was it helpful for your dog?

\subsection{Hormone assays}

Saliva tubes were stored at $-20^{\circ} \mathrm{C}$ until analysis. Before analyses, the saliva tubes were thawed and centrifuged for $10 \mathrm{~min}$ at $1000 \mathrm{xg}$. Salivary cortisol was analysed in duplicates with a commercial radio-immunoassay kit (Spectria Cortisol RIA, Orion Diagnostica, Turku, Finland). The quantitation limit of the cortisol analysis was $0.36 \mathrm{ng} / \mathrm{ml}$. The intra-assay coefficient of variation was $11.0 \%$. To eliminate inter-assay variation, all samples were measured in the same assay. All samples had cortisol concentrations above the quantitation limit.

Urine samples were cooled immediately after collection in an ice-bath and centrifuged for $10 \mathrm{~min} 1300 \mathrm{xg}$ at $4{ }^{\circ} \mathrm{C}$ and stored at $-80^{\circ} \mathrm{C}$. Samples were first purified with a solid phase extraction (SPE). SPE columns (Oasis HLB 1 cc Vac Cartridge, $30 \mathrm{mg}$, Waters Corporation, Milford, U.S.A.) were conditioned with $1 \mathrm{ml}$ methanol and $1 \mathrm{ml}$ water and then $1 \mathrm{ml}$ of urine sample with $10 \mu \mathrm{l}$ of phosphoric acid was added to the columns. Columns were washed with $1 \mathrm{ml} \mathrm{10 \%}$ acetonitrile containing $0.1 \%$ trifluoroacetic acid (TFA) in water and eluted with $1 \mathrm{ml}$ 80:20 (acetonitrile:water). Eluted samples were evaporated to dryness with a stream of nitrogen at $37^{\circ} \mathrm{C}$ and stored at $-80^{\circ} \mathrm{C}$ until oxytocin analysis.

Oxytocin was anlysed with an enzyme-linked immunosorbent assay kit (Oxytocin ELISA kit, Enzo Life Sciences, Lausen, Switzerland). Before ELISA, SPE-purified and dried urine samples were reconstituted with $250 \mu$ l assay buffer supplied in the ELISA kit and analysed in duplicate according to the assay kit instructions. The inter- and intra-assay coefficients of variations were $9.9 \%$ and $12.5 \%$, respectively. The recovery of the assay was tested by adding a known amount of oxytocin to urine. Recovery for urine samples was 102.0 (13.7) \%, mean (standard deviation), $\mathrm{n}=12$. The quantitation limit of the urinary oxytocin was $3.9 \mathrm{pg} / \mathrm{ml}$ and all samples were above the quantitation limit.

The urinary oxytocin concentrations were normalized by measuring creatinine concentrations in every urine sample to control variations in urine flow. The creatinine levels were measured with an enzymatic Konelab method by automatic analyser Konelab 30i (Thermo Fisher Scientific, Vantaa, Finland). Urinary oxytocin concentrations are presented here as oxytocin to creatinine ratio, $\mathrm{pg} / \mathrm{mg} \mathrm{Cr}$.

\subsection{Missing data}

All four saliva samples for cortisol analyses were successfully collected from 20 dogs, and the two urine samples for oxytocin were successfully collected from 24 dogs. Most missing samples were due to either the dog not urinating, or not producing enough saliva. In addition, one dog behaved aggressively during saliva sampling, and the sampling was not continued. Videos of 28 dogs were analysed, of which 25 participated in all tests. Missing data was due to one dog being too fearful to participate into the noise test, and two dogs leaving the study before finishing all three test days. Altogether we had the entire data from behavioural and physiological measures from 20 dogs.

\subsection{Statistics}

The number of crossed squares during each interval was calculated. The duration the dog spent near their owner during each interval was defined as the sum of duration the dog spent in squares 1 and 5 (Fig. 3). The data for number of crossed squares was lntransformed to obtain an approximate normal distribution. The differences between the treatments in number of crossed squares and time spent near their owner were tested with repeated measures linear mixed models. The models included test day (1st, 2nd or 3rd), treatment (CONTROL, LIGHT, DEEP), interval (pre-noise, noise and recovery) and order of treatments (1,2 or 3 ) as fixed effects, as well as any statistically significant two-way interactions between these effects. Dog nested within order of treatments was used as a random factor.

The effect of vest type on saliva cortisol was tested with a linear mixed model with saliva sample (COR2, COR3, COR4) as repeated factor and saliva sample at arrival (COR1) as a covariate.

Results of the linear mixed models are presented as estimated means with the standard error of mean (SEM) except for the number of squares crossed which is presented as an estimated mean with a $95 \%$ confidence interval $(\mathrm{CI})$ due to reconversion of the logarithmic variable.

For the statistical analyses total duration was calculated for body and tail postures and panting and number of bouts of yawning, licking and fence manipulation per each of the test intervals (pre-noise, noise, recovery). The data failed to follow normal distribution, even after attempting transformation, and the differences between the three treatments during each interval with related samples were tested by Wilcoxon Signed-rank tests, and if found significant, pair-wise comparisons were performed with Friedman tests. Also differences between OXY1 and OXY2 were tested with a Friedman test. Results from the non-parametric tests are reported as medians and interquartile ranges (IQR).

Spearman rank correlation tests were used to test for associations between oxytocin and cortisol samples, and between the times the dog spent near their owner, the behavioural indexes derived from the questionnaire (Table 4) and dogs lying time during the noise intervals. 
Table 4

Spearman rank correlation coefficients $\left(\mathrm{r}^{\mathrm{s}}\right)$ between lying duration of noise-phobic pet dogs during two 2-min noise interval with either DEEP $(10-12 \mathrm{~mm} \mathrm{Hg})$, LIGHT pressure vest $(2-3 \mathrm{~mm} \mathrm{Hg}$ ) or CONTROL (no vest), and general fearfulness and noise fear frequency (questionnaire-derived variables from Tiira and Lohi, 2014, see Table 3 for details).

Questionnaire-derived Lying duration during the noise interval. Dogs were

behavioural variables wearing either a DEEP pressure vest, a LIGHT pressure vest or no vest at all (CONTROL).

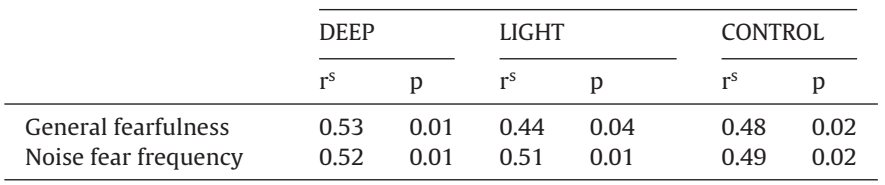

\section{Results}

\subsection{Behaviour during the test}

We found a difference between the treatments in median lying time during the noise interval (Wilcoxon sign, $\mathrm{p}<0.02$ ), and pairwise comparisons revealed thatthe significant difference was between the DEEP and CONTROL treatments (Friedman test $\mathrm{p}<0.03$ ). The median (IQR) lying duration of dogs was shorter when dogs were wearing the DEEP, vest during the noise interval, compared to CONTROL and no difference was found compared to the LIGHT; 0 (66.90) s vs. 39.00(109.00) s and 12.70(110.10) s, respectively.

Overall the dogs spent (mean \pm sem) $33 \pm 4.5$ s near their owners during the 6 min test period. This duration was not affected by the treatment nor the interval. However, we found an interval*treatment interaction showing that dogs in the CONTROL treatment spent less time near their owner during the noise interval than during the pre-noise or recovery intervals ( $\mathrm{p}<0.05$ for both). No difference was found between intervals within the LIGHT or DEEP treatments (Fig. 5).

Treatment or test day had no effect on the mean (CI95\%) number of crossed squares; which was overall 2.1 (1.2-3.1). Intervals did differ $(p<0.001)$ : Mean number of squares the dogs crossed reduced from pre-noise interval to noise and recovery intervals: from $3.8(1.0-1.2)$ to $2.4(0.8-1.0)$ and $0.6(0.3-0.5)$, respectively $(p<0.02$ for all). We also found an interaction between the test day and intervals $(p<0.001)$ : dogs crossed more squares during the noise interval of the 1 st test day than during the 2nd or the 3rd days ( $p<0.01$ for both, Fig. 6 ). No test day effect was found for the other intervals, nor was a treatment*test day interaction detected.

We found no treatment, interval or day effects on any of the other behaviours recorded ( $p>0.05$ for all).

\subsection{Correlations between behaviours observed during the tests and fearful behaviour at home}

We found a positive correlation between the time spent near the owner and the noise fear frequency ( $\mathrm{p}<0.001, \mathrm{rs}=0.75, \mathrm{~N}=24$ ), but only when the dogs were wearing the DEEP vest during the noise interval. We also found a positive correlation between the time spent near the owner during the recovery interval, and the noise fear frequency $(\mathrm{p}<0.001, \mathrm{rs}=0.62, \mathrm{~N}=24)$ and noise reactivity index $(\mathrm{p}<0.04, \mathrm{rs}=0.43, \mathrm{~N}=24)$, when the dogs were wearing the DEEP pressure vest.

We found positive correlations between lying duration during the noise interval in every treatment and general fearfulness and noise fear frequency ( $\mathrm{p}<0.05$ for all, Table 4.)

There was no difference in cortisol level between treatments or test days. However, test intervals did differ: the cortisol level was higher in the COR3 sample than in the COR2 or COR4 sam-
Table 5

Spearman rank correlation coefficients $\left(\mathrm{r}^{\mathrm{s}}\right)$ between canine urine oxytocin, sampled after wearing a DEEP pressure vest $(10-12 \mathrm{~mm} \mathrm{Hg}$ ) for $40 \mathrm{~min}$, and general fearfulness, noise fear frequency and reactivity index (questionnaire-derived behavioural variables from Tiira and Lohi, 2014, see Table 3 for details, $\mathrm{N}=23$ ).

\begin{tabular}{lll}
\hline $\begin{array}{l}\text { Questionnaire-derived } \\
\text { behavioural variables }\end{array}$ & \multicolumn{2}{l}{$\begin{array}{l}\text { Urine-oxytocin in dogs } \\
(\mathrm{N}=23)\end{array}$} \\
\cline { 2 - 3 } & $\mathrm{rs}$ & $\mathrm{p}$ \\
\hline General fearfulness & 0.50 & 0.016 \\
Noise fear frequency & 0.47 & 0.024 \\
Reactivity index & 0.56 & 0.005 \\
\hline
\end{tabular}

ples $(4.94+/-0.44 \mathrm{ng} / \mathrm{ml}$ vs. $4.33+/-0.43 \mathrm{ng} / \mathrm{ml}$ and $4.04+/-$ $0.45 \mathrm{ng} / \mathrm{ml}, \mathrm{p} \leq 0.05$ for all, respectively, $\mathrm{N}=20$ ).

Median (IQR) urine OXY1 and OXY2 levels did not differ; 20.1 (10.31) pg/mg Cr and 18.0 (12.01) pg/mg Cr, respectively. And they had a strong positive inter-correlation ( $\mathrm{rs}=0.813, \mathrm{p}<0.001, \mathrm{~N}=24$ ). No statistically significant correlation was found between OXY and COR samples.

Time spent near the owner during the recovery interval during the DEEP treatment correlated positively with OXY1 and OXY2 ( $\mathrm{rs}=0.44, \mathrm{p}<0.03$ and $0.50, \mathrm{p}<0.01$, respectively, $\mathrm{N}=24$ for both).

Total time spent lying down during the noise interval with DEEP or LIGHT pressure vest correlated positively with the (COR3) postnoise saliva cortisol ( $\mathrm{rs}=0.57$ and 0.49 , respectively, $\mathrm{p}<0.05$ for both).

We found no significant associations between cortisol levels and questionnaire derived behavioural variables ( $p>0.05$ for all). However, we did find significant positive associations between OXY2 and general fearfulness, noise fear frequency and reactivity index $(\mathrm{p}<0.02$ for all, Table 5).

\subsection{Follow-up questionnaires}

According to the answers $(\mathrm{N}=28)$ to the first follow-up questionnaire the pressure vest had been used in daily life by 17 dogs. The experience of the use of the pressure vest was mainly positive (14/17-82\%), or neutral (3/17-18\%).

Regarding New Year's Eve, when dogs are usually exposed to fireworks, we got 26 answers to the second follow up questionnaire. Most of the dogs (20) had used pressure vest on New Year's Eve. These owners reported mainly positive experiences (16/20-80\%) or neutral experiences (4/20-20\%).

\section{Discussion}

We did not observe large differences in behaviour when using a pressure vest in noise phobic dogs in a double-blinded experimental setup. However, some subtle, but interesting, differences in behaviour were observed. Dogs were lying down less during the noise interval when wearing the DEEP pressure vest compared to dogs without vest. We did not subjectively classify the valence (negative or positive) of observed behaviours in this study, but based on our results, increased lying duration is most likely a fearrelated behaviour in this setup. Lying duration during noise interval was positively correlated to saliva cortisol when wearing either vest. In addition, we found that the strength of noise fear in the home environment, as assessed by the owners, was also positively correlated to lying duration during the noise interval. Similarly, freezing behaviour, has also been shown to be common behavioural response to noise interval (Araujo et al., 2013; Gruen et al., 2015; Tiira et al. in Press)

Wearing the DEEP vest increased the time the dogs spent near their owner during the noise and recovery intervals. The dogs without vest spent less time near their owner during the noise test than 


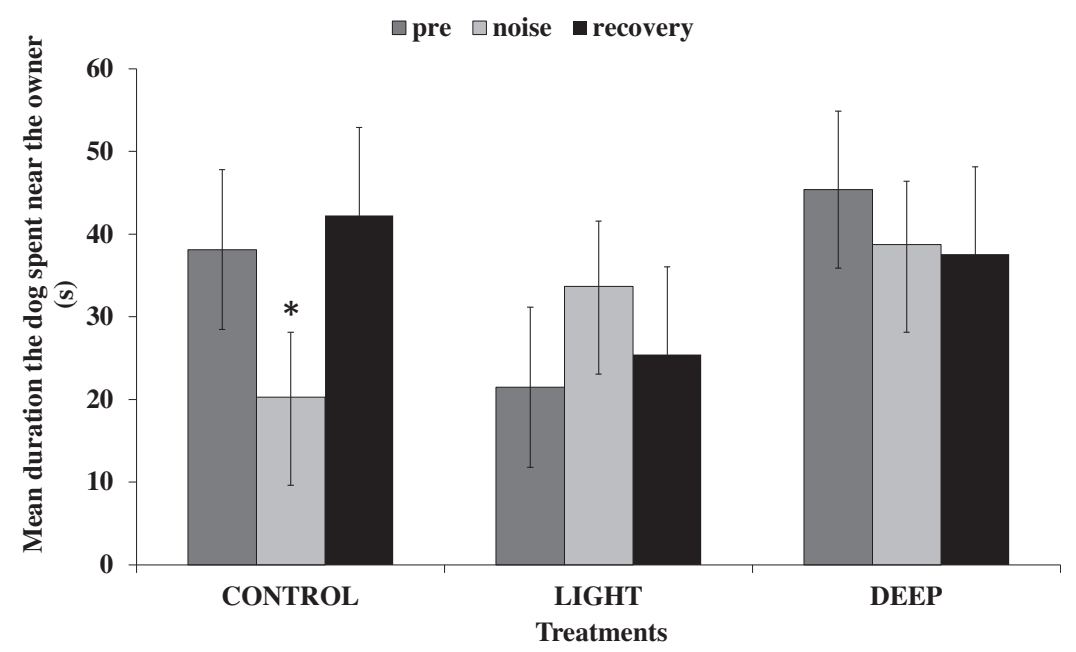

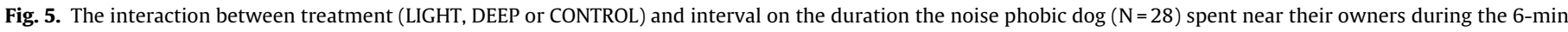
test. Each interval (pre, noise and recovery) lasted for two minutes.

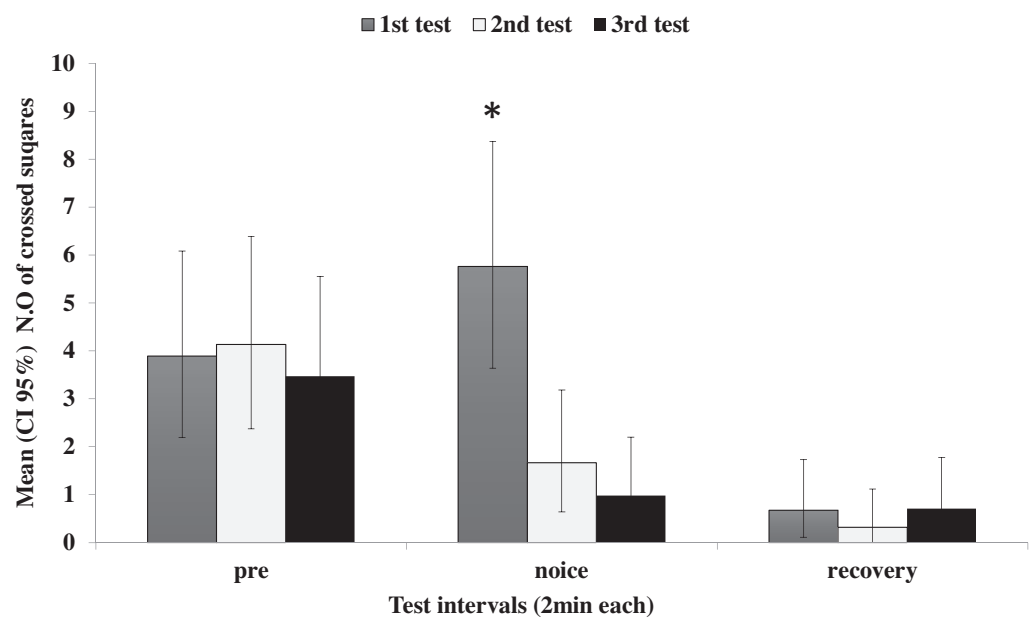

Fig. 6. Interaction between the test sequences and intervals on the mean number squares the noise-phobic dogs $(\mathrm{N}=28)$ crossed.

pre-noise and recovery intervals. In addition, the strength of the noise fear of dogs at home, as assessed by their owners, correlated positively with the duration the dogs spent near their owner during the noise interval and during the recovery interval, but only when wearing the DEEP pressure vest. In fearful situations, dogs are especially dependent on their owners (Kerepesia et al., 2015) and it is possible that in very fearful dogs, the pressure vest facilitates such behaviour. As the time spent near the owner when wearing the DEEP pressure vest during recovery was correlated to oxytocin, and as oxytocin level was higher also in dogs with a higher level of fear in home conditions, it is possible that this owner seeking behaviour is mediated by oxytocin. Oxytocin is related to social bonding (Nagasawa et al., 2015) and even short-term interaction between dogs and their owners will raise oxytocin levels (Handlin et al., 2011). Dogs have been shown to show more affiliation to their owners after nasal oxytocin spray than before (Romero et al., 2013). However, we were not able to show an effect of the vest on the oxytocin level during the vest fitting session, thus we cannot conclude that the vest-induced increase of owner-seeking was due to oxytocin, and the topic warrants further investigation.

We found that baseline urine oxytocin correlated positively with saliva cortisol after the noise test. It is possible that by preventing physical and eye contact between dog and the owner during the test, we induced an additional stress factor. This might have been especially stressful for dogs with high baseline oxytocin levels, as these might be dogs with a higher motivation to seek owner comfort (Romero et al., 2013). On the other hand, due to the positive correlation between oxytocin and fearfulness at home, it is also possible that this is merely due to these dogs being more fearful in general, and thus more effected by the test. Dogs have large between-individual behavioural variation in how they react in fearful or stressful situations (Araujo et al., 2013; Gruen et al., 2015; Tiira et al. In press) and it is therefore difficult to classify one or two behaviours which would most likely indicate a fearful emotional state. Therefore, in this study we decided to measure only the difference in the observed behaviour with or without a vest. The strength of this method is objectivity, however we cannot gain any information on the valence or arousal of emotions using this method. The other option would have been to subjectively assess the level of fearful emotion for example by using several dog experts, which would have reduced the objectivity, but allowed assessment of valence and arousal of emotion.

The noise interval was a stressful situation for the dogs in this study, indicated by changes both in behaviour (reduced crossed squares) due to the noise, and an increased cortisol level after the test as compared to just being in the test room. This indicated our experimental setup has been successful in inducing fear. A similar rise in cortisol after noise interval was also reported by 
Hydbring-Sandberg et al. (2004). The behaviour of the dogs during the noise interval was different on the first test day than during the following two, with more crossed squares on the first test day. This also could be due to habituation during the experiment. This experiment indicate that the most fearful dogs actually became less active, i.e. showed increased freezing behaviour, as a results of increased stress. This might also indicate an increased fear level in the dogs during the consecutive test days, as reported also by Araujo et al. (2013) and Gruen et al. (2015).

The fact that the majority (approximately $80 \%$ ) of owners had positive experiences of the vest, when used in the normal environment of the dog, indicates that the vest does have a therapeutic effect on some dogs, even though this was not clearly shown in the current experimental set-up. This is a similar result to that of Cottam et al. (2013), testing another type of pressure clothing on dogs with thunderstorm phobia. It needs to be remembered that in an artificial test situation the owner behaviour is difficult to standardize, and not all dogs react similar to same noise stimuli. According to some owners (results not reported), their dogs did not react similarly in our artificial tests situation compared with real thunder/fireworks (i.e. were not fearful), suggesting that these dogs might have not experienced this situation as "real". However, as owners hoping for help for their dogs might over interpret changes in the dogís behaviour due to the vest (Cottam et al., 2013), and as owners might not always recognize subtle signs of stress (Maritia et al., 2012), recordings in the home-environment should be objective and preferably blinded.

Noise phobia is a very heterogeneous symptom, with dogs reacting to various sounds, and with a large range of severity (Storengen and Lingaas, 2015). In addition, dogs show individual variation in their reaction to stressful situations (Horváth et al., 2007). Thus the study population should be rather large to find possible therapeutic effects, as well as features describing those dogs that benefit from any tested treatment. We suggest that future studies investigating the therapeutic effect of pressure vests should include a larger study population, a real loud noise situation, and assessment of valence and arousal of emotion. Also combining pressure vests to behavioural therapy would be worth studying.

\section{Conclusions}

The pressure vest changed the behaviour of dogs during a noise interval in a double-blinded, controlled experiment, but only to a limited extent. The vest facilitated dogs to seek comfort from their owners and reduced the time dogs spent freezing.

\section{Acknowledgements}

The authors thank all the dog owners and their dogs for volunteering to participate in the study and Lymed ${ }^{\circledR}$ Oy for providing the pressure vests used. We like to thank the Alma and K. A. Snellman Foundation, Oulu, Finland, the Finnish Foundation of Veterinary Research, ERCStG and Lymed ${ }^{\circledR} \mathrm{Oy}$ for the financial support.

\section{References}

Araujo, J.A., de Rivera, C., Landsberg, G.M., Adams, P.E., Milgram, N.W., 2013. Development and validation of a novel laboratory model of sound-induced fear and anxiety in Beagle dogs. J. Vet. Behav. 8, 204-212, http://dx.doi.org/10. 1016/j.jveb.2012.10.008.

Bello, D., Redlich, C.A., Stowe, M.H., et al., 2008. Skin exposure to aliphatic polyisocyanates in the auto body repair and refinishing industry: II. A quantitative assessment. Ann. Occup. Hyg. 52, 117-124, http://dx.doi.org/10. 1093/annhyg/mem066.

Blackwell, E., Bradshaw, J., Casey, R., 2013. Fear responses to noises in domestic dogs: prevalence, risk factors andco-occurrence with other fear related behaviour. Appl. Anim. Behav. Sci. 145, 15-25, http://dx.doi.org/10.1016/j. applanim.2012.12.004.
Blairs, S., Slater, S., Hare, D.J., 2007. The clinical application of deep touch pressure with a man with autism presenting with severe anxiety and challenging behaviour. Br. J. Learn. Disabil. 35, 214-220, http://dx.doi.org/10.1111/j.14683156.2007.00452.x.

Carter, C., 2003. Developmental consequences of oxytocin. Physiol. Behav. 79, 383-397, http://dx.doi.org/10.1016/S0031-9384(03)00151-3.

Cottam, N., Dodman, N.H., Ha, J.C., 2013. The effectiveness of the anxiety wrap in the treatment of canine thunderstorm phobia: an open-label trial. J. Vet. Behav. 8, 154-161, http://dx.doi.org/10.1016/j.jveb.2012.09.001.

Dale, A.R., Walker, J.K., Farnworth, M.J., Morrissey, S.V., Waran, N.K., 2010. A survey of owners' perceptions of fear of fireworks in a sample of dogs and cats in New Zealand. N. Z. Vet. J. 58, 286-291, http://dx.doi.org/10.1080/00480169.2010. 69403.

Edelson, S.M., Edelson, M.G., Kerr, D.C., Grandin, T., 1999. Behavioral and physiological effects of deep pressure on children with autism: a pilot study the efficacy of Grandinin's Hug machine. J. Occup. Ther. 53, 145-152, http://dx. doi.org/10.5014/ajot.53.2.145.

Grandin, T., 1992. Calming effects of deep touch pressure in patients with autistic disorder, college students, and animals. J. Child Adolesc. Psychopharmacol. 2, 63-72, http://dx.doi.org/10.1089/cap.1992.2.63.

Gruen, M.E., Case, B.C., Foster, M.L., Dorman, D.C., Sherman, B.L., 2015. The use of an open-field model to assess sound-induced fear and anxiety-associated behaviors in Labrador retrievers. J. Vet. Behav. 10, 338-345, http://dx.doi.org/ 10.1016/j.jveb.2015.03.007.

Hänninen, L., Pastell, M., 2009. CowLog: open-source software for coding behaviors from digital video. Behav. Res. Methods 41, 472-476, http://dx.doi.org/10. 3758/BRM.41.2.472.

Handlin, L., Hydbring-Sandberg, E., Nilsson, A., Ejdebäck, M., Jansson, A., Uvnäs-Moberg, K., 2011. Short-term interaction between dogs and their owners: effects on oxytocin, cortisol, insulin and heart rate-an exploratory study. anthrozoos: a multidisciplinary. J. Interact. People Anim. 24, 301-315, http://dx.doi.org/10.2752/175303711 X13045914865385.

Horváth, Z., Igyártór, B.Z., Magyar, A., Miklósi, A., 2007. Three different coping styles in police dogs exposed to a short-term challenge. Horm. Behav. 52, 621-630, http://dx.doi.org/10.1016/j.yhbeh.2007.08.001.

Hydbring-Sandberg, E.W., von Walter, L., Höglund, K., Svartberg, K., Swenson, L., Forkman, B., 2004. Physiological reactions to fear provocation in dogs. J. Endocrinol. 180, 439-439 http://joe.endocrinology-journals.org/content/180/ 3/439.long.

Kerepesia, A., Dókaa, A., Miklósib, A., 2015. Dogs and their human companions: the effect of familiarity on dog-human interactions. new directions in canine behavior dogs and their human companions. Behav. Process. 110, 27-36, http://dx.doi.org/10.1016/j.beproc.2014.02.005.

King, C., Buffington, L., Smith, T.J., Grandin, T., 2014. The effect of a pressure wrap (ThunderShirt $^{\circledR}$ ) on heart rate and behavior in canines diagnosed with anxiety disorder. J. Vet. Behav. 9, 215-221, http://dx.doi.org/10.1016/j.jveb.2014.06. 007.

Maritia, C., Gazzanoa, A., Lansdown Mooreb, J., Baraglia, P., Chellia, L., Sighieria, C., 2012. Perception of dogs' stress by their owners. J. Vet. Behav. 7, 213-219, http://dx.doi.org/10.1016/j.jveb.2011.09.004.

McCobb, E.C., Brown, E.A., Damiani, K., Dodman, N.H., 2001. Thunderstorm phobia in dogs: an Internet survey of 69 cases. J. Am. Anim. Hosp. Assoc. 37, 319-324, http://dx.doi.org/10.5326/15473317-37-4-319.

Mills, D., 2005. Management of noise fears and phobias in pets. In Practice, Volume 27, pp. 248-255. 10.1136/inpract.27.5.248.

Mullen, B., Champagne, T., Krishnamurty, S., Dickson, D., Gao, R.X., 2008. Exploring the safety and therapeutic effects of deep pressure stimulation using a weighted blanket. Occup. Ther. Mental Health 24, 65-89, http://dx.doi.org/10. 1300/J004v24n01_05.

Nagasawa, M., Mitsui, S., En, S., Ohtani, N., Ohta, M., Sakuma, Y., Onaka, T., Mogi, K., Kikusui, T., 2015. Oxytocin-gaze positive loop and the coevolution of human-dog bonds. Science 348, 333-336, http://dx.doi.org/10.1126/science. 1261022.

Odendaal, J.S.J., Meintjes, R.A., 2003. Neurophysiological correlates of affliative behaviour between humans and dogs. Vet. J. 165, 296-301, http://dx.doi.org/ 10.1016/S1090-0233(02)00237-X.

Overall, K.L., 2000. Natural animal models of human psychiatric conditions: assessment of mechanism and validity. Prog. Neuropsychopharmacol. Biol. Psychiatry 24, 727-776, http://dx.doi.org/10.1016/S0278-5846(00)00104-4.

Overall, K.L., 2013. Manual of Clinical Behavioral Medicine for Dogs and Cats, 1st ed. Elsevier.

Panksepp, J., 1992. Oxytocin effects on emotional processes: separation distress, social bonding and relationships to psychiatric disorders. Ann. N. Y. Acad. Sci. 652, 243-252, http://dx.doi.org/10.1111/j.1749-6632.1992.tb34359.x.

Pedersen, C.A., Caldwell, J.D., Drago, F., Noonan, L.R., Peterson, G., Hood, L.E., 1988. Grooming behavioral effects of oxytocin Pharmacology, ontogeny, and comparisons with other nonapeptides. Ann. N. Y. Acad. Sci. 525, 245-256, http://dx.doi.org/10.1111/j.1749-6632.1988.tb38610.x.

Pedersen, W.C., Gonzales, C., Miller, N., 2000. The moderating effect of trivial triggering provocation on displaced aggression. J. Pers. Soc. Psychol. 78, 913-927, http://dx.doi.org/10.1037/0022-3514.78.5.913.

Romero, T., Nagasawa, M., Mogi, K., Hasegawa, T., Kikusui, T., 2013. Oxytocin promotes social bonding in dogs. PNAS 111, 9085-9090, http://dx.doi.org/10. 1073 /pnas.1322868111.

Sherman, B.L., Mills, D.S., 2008. Canine anxieties and phobias: an update on separation anxiety and noise aversions. Practical applications and new 
perspectives in veterinary behavior. Vet. Clini. North Am. 38, 1081-1106, http://dx.doi.org/10.1016/j.cvsm.2008.04.012.

Storengen, L.M., Lingaas, F., 2015. Noise sensitivity in 17 dog breeds: prevalence, breed risk and correlation with fear in other situations. Appl. Anim. Behav. Sci. 171, 152-160, http://dx.doi.org/10.1016/j.applanim.2015.08.020.

Tiira, K., Lohi, H., 2015. Early life experiences and exercise associate with canine anxieties. PLoS One 10, http://dx.doi.org/10.1371/journal.pone.0141907.

Tiira, K., Lohi, H., 2014. Reliability and validity of a questionnaire survey in canine anxiety research. Appl. Anim. Behav. Sci. 155, 82-92, http://dx.doi.org/10. 1016/j.applanim.2014.03.007.
Tiira K, Sulkama S, Lohi H., Prevalence, co-morbidity and behavioral variation in canine anxiety. J. Vet. Behav., Accepted.

Uvnäs-Moberg, K., 2006. Physiological and endocrine effects of social contact. Ann. N. Y. Acad. Sci. 15, 146-163, http://dx.doi.org/10.1111/j.1749-6632.1997. tb51917.x.

van der Waaij, E.H., Wilsson, E., Strandberg, E., 2008. Genetic analyses of results of a Swedish behavior test on German Shepherd Dogs and Labrador Retrievers. J. Vet. Behav. Clin. Appl. Res. 3, 2853-2861, http://dx.doi.org/10.2527/jas.20070616. 